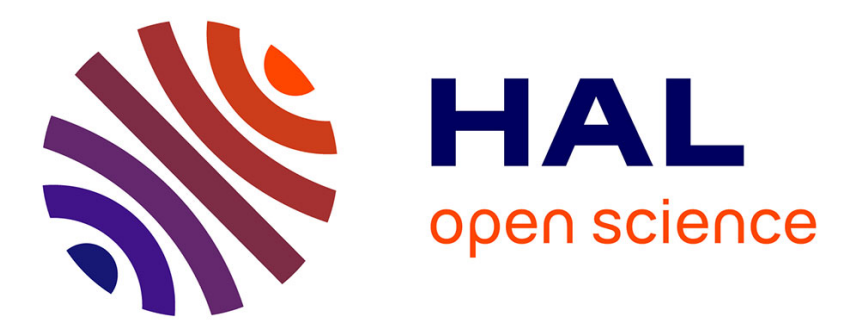

\title{
Nonlinear Self-Confined Plasmonic Beams: Experimental Proof
}

Tintu Kuriakose, Gilles Renversez, Virginie Nazabal, Mahmoud M. R. Elsawy, Nathalie . Coulon, Petr Němec, Mathieu Chauvet

\section{> To cite this version:}

Tintu Kuriakose, Gilles Renversez, Virginie Nazabal, Mahmoud M. R. Elsawy, Nathalie . Coulon, et al.. Nonlinear Self-Confined Plasmonic Beams: Experimental Proof. ACS photonics, 2020, 7 (9), pp.2562-2570. 10.1021/acsphotonics.0c00906 . hal-02961176

\section{HAL Id: hal-02961176 https://hal-univ-rennes1.archives-ouvertes.fr/hal-02961176}

Submitted on 19 Oct 2020

HAL is a multi-disciplinary open access archive for the deposit and dissemination of scientific research documents, whether they are published or not. The documents may come from teaching and research institutions in France or abroad, or from public or private research centers.
L'archive ouverte pluridisciplinaire HAL, est destinée au dépôt et à la diffusion de documents scientifiques de niveau recherche, publiés ou non, émanant des établissements d'enseignement et de recherche français ou étrangers, des laboratoires publics ou privés. 


\section{Nonlinear Self-Confined Plasmonic Beams: Experimental Proof}

Tintu Kuriakose, Gilles Renversez,* Virginie Nazabal, Mahmoud M. R. Elsawy, Nathalie Coulon, Petr Němec, and Mathieu Chauvet*

Cite This: ACS Photonics 2020, 7, 2562-2570

Read Online

ACCESS | Lill Metrics \& More | 回 Article Recommendations | St Supporting Information

ABSTRACT: Controlling low power light beam self-confinement with ultrafast response time opens up opportunities for the development of signal processing in microdevices. The combination of a highly nonlinear medium with the tight confinement of plasmonic waves offers a viable but challenging configuration to reach this goal. In the present work, a beam propagating in a plasmonic structure that undergoes a strongly enhanced selffocusing effect is reported for the first time. The structure consists of a chalcogenide-based four-layer planar geometry engineered to limit plasmon propagation losses while exhibiting efficient Kerr self-focusing at moderate power. As expected from theory, only TM-polarized waves exhibit such a behavior. Different experimental arrangements are tested at telecom wavelengths and

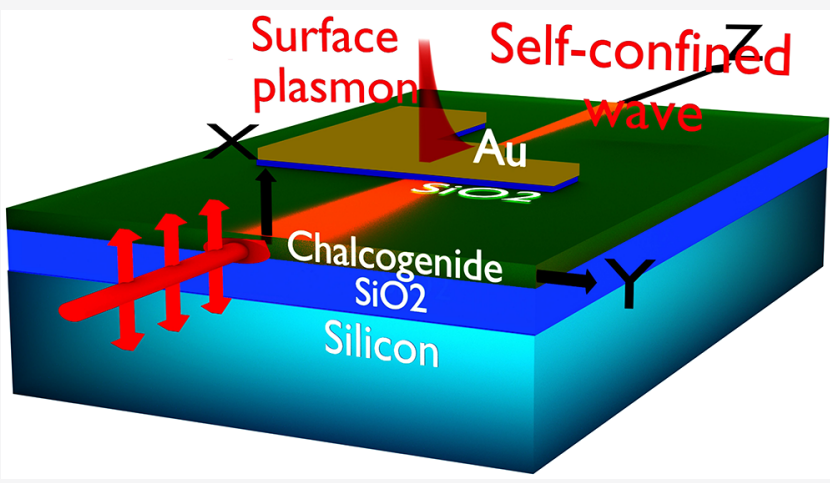
compared with simulations obtained from a dedicated model. The observed efficient beam reshaping takes place over a distance as low as $100 \mu \mathrm{m}$, which unlocks new perspectives for the development of integrated photonic devices.

KEYWORDS: nonlinear integrated plasmonics, self-confined waves, optical Kerr effect, chalcogenide glass, planar waveguides, modeling

$\mathrm{T}$ he efficiency of nonlinear optical processes is well-known to benefit from intense light. One way to achieve such high electromagnetic fields at a low excitation level is to exploit light interactions with metal nanostructures. In that sense, the surface plasmon polariton $(\mathrm{SPP})^{1}$ at a metal-dielectric interface, associated with both a collective oscillation of free charges in the metal and an extremely confined light wave, has been a key discovery. Since then, plasmonics has evolved into a flourishing research field ${ }^{2}$ thanks notably to the numerous developments on metamaterials. ${ }^{3,4}$ It was then natural to combine plasmonics and nonlinear optics to envision a variety of fascinating and original physical phenomena with great potential applications. Nonlinear plasmonics ${ }^{5}$ has thus grown significantly in recent years. Among other nonlinear phenomena, self-trapped beams, that can lead to spatial solitons, ${ }^{6}$ have already been observed at very low optical power in nonstandard nonlinear media such as photorefractive ${ }^{7,8}$ or liquid crystal $^{9}$ but their slow formation times are not suitable for ultrafast signal processing. To the contrary Kerr spatial solitons ${ }^{10,11}$ have a fast response but necessitates high optical power. Self-trapped plasmonic waves formed in Kerr-like nanostructures should authorize the use of lower power by exploiting enhanced nonlinear effects while allowing nonlinear optical components to be scaled down in size. The main possible uses of these nonlinear plasmonic waves would be in integrated optics devices where high speed, small footprint, and reduced power consumption are key factors. ${ }^{12,13}$
First descriptions of one-dimensional nonlinear self-trapped waves with potential to form plasmon-solitons at metal/ dielectric interfaces and nonlinear surface waves at dielectric/ dielectric interfaces were unveiled in the early eighties. ${ }^{14-17}$ The concept being to propagate a plasmon-polariton wave at a metal-nonlinear material interface in order to induce an exalted Kerr self-focusing effect giving rise to a self-trapped wave. Up to now, the problem has been tackled only theoretically, based on numerous analytical and numerical calculations. $^{18-28}$ Despite this constant and comprehensive modeling development, no experimental evidence of selftrapped nonlinear plasmonic waves, including their stable forms, so-called plasmon-solitons in ref 18, has yet been revealed, despite almost four decades since the first publications on nonlinear surface waves. ${ }^{14,15}$ Among the practical challenges inherent to this plasmon assisted selffocusing demonstration are the large propagation losses associated with plasmons, the request for a strong Kerr

Received: June 4, 2020

Published: August 17, 2020 


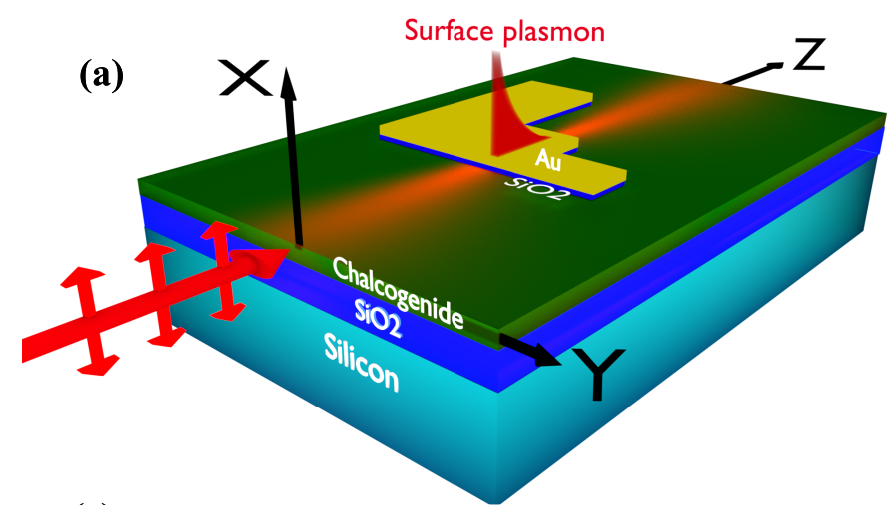

(c)

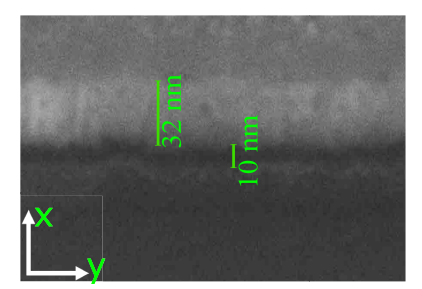

(d)

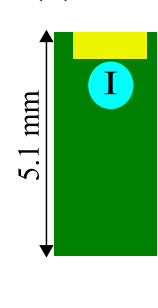

exit face

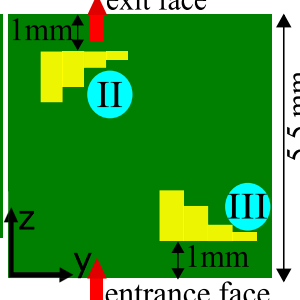

(b)

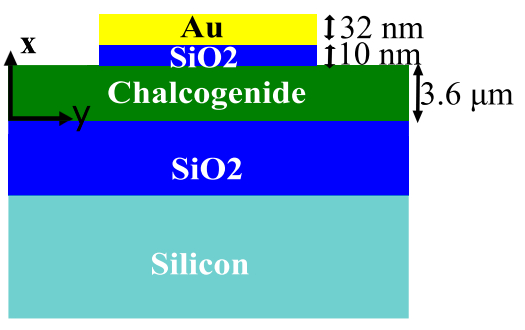

(e)

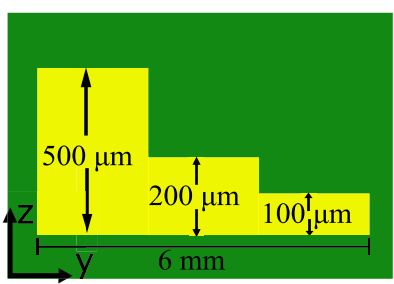

Figure 1. Description of the samples. (a) Artistic 3-D view of a sample. (b) Description of the cross section of the plasmonic structure. (c) SEM image of the $\mathrm{Au} / \mathrm{SiO}_{2} /$ chalcogenide interfaces. (d) Schematic overall top view of the samples showing the three studied configurations denoted by I, II, and III. (e) Detailed schematic top view of a staircase metallic pattern.

coefficient, and the limitations due to the damage threshold intensity of the plasmonic structure.

In the present work, a four-layer slab component is designed and fabricated to support a SPP with moderate losses along with a strong Kerr nonlinear effect. This structure allows the first experimental observation of a self-confined nonlinear wave that combines a self-trapped wave and a SPP in a single wave. This nonlinear wave reveals a strongly enhanced self-focusing occurring over distances as short as $100 \mu \mathrm{m}$ due to the plasmonic effect. The content of this Article is as follows: first, the structure design and fabrication steps are described. Second, nonlinear optical characterizations are presented. Third, the dedicated nonlinear propagation model is described. Finally, simulations are confronted to the experiments, and results are discussed.

\section{DESIGN AND FABRICATION OF THE STRUCTURES}

A chalcogenide glass is chosen as the nonlinear medium since it exhibits a Kerr effect about 2 orders of magnitude stronger than silica thanks to the strong polarizability of the chalcogen elements while offering a fast temporal response with a femtosecond time scale due to its underlying mechanism based on electronic polarization. ${ }^{29}$ The origin of our nonlinear plasmonic component is a planar chalcogenide waveguide that possesses the suitable linear and nonlinear properties to form Kerr spatial solitons at near-infrared wavelengths. ${ }^{30,31}$ A metal film is added for the propagating wave to trigger a plasmonic effect. However, a compromise has to be found between a tight light confinement provided by the plasmonic effect, which we want to exploit to efficiently exalt the Kerr effect, and the high propagation losses induced by the metal that would prevent the observation of the Kerr self-focusing.

The chosen solution is to insert a thin silica buffer layer between the metal and the nonlinear dielectric layer. The designed waveguide including its plasmonic structure (PS) derives from our previously published theoretical studies ${ }^{21,22}$ and from Finite Element Method (FEM) simulations (see section 3 of the Supporting Information). To be more specific, the Kerr layer is a $3.6 \mu$ m thick $\mathrm{Ge}_{28.1} \mathrm{Sb}_{6.3} \mathrm{Se}_{65.6}$ amorphous chalcogenide film ${ }^{32}$ (Figure 1a,b and Supporting Information). This glass with a $2.16 \mathrm{eV}$ bandgap and with a refractive index of 2.54 at $1.55 \mu \mathrm{m}$ was chosen due to its large Kerr nonlinearity $\left(n_{2}=5.5 \times 10^{-18} \mathrm{~m}^{2} / \mathrm{W}\right)$, moderate two-photon absorption coefficient $\left(\alpha_{2}=0.43 \mathrm{~cm} / \mathrm{GW}\right)$, a high damage threshold intensity evaluated to approximately $2.5 \mathrm{GW} /$ $\mathrm{cm}^{2},{ }^{31,33}$ and a low photosensitivity. ${ }^{33}$ Note that the twophoton absorption at $1.55 \mu \mathrm{m}$ is attributed to an Urbach's absorption tail related to both thermal effects and static disorder. ${ }^{33}$ A silica buffer layer followed by a gold layer are then deposited on top of the chalcogenide material.

FEM simulations ${ }^{34,35}$ have been performed to compute the nonlinear modes propagating in the structure in order to determine the optimum thicknesses for these top layers. It is important to note that the modes are calculated in nonlinear regime, that is, their amplitude is dependent on the magnitude of the self-focusing effect. These simulations showed that the silica buffer layer thickness $d$ is the most critical parameter for the design, as expected from a previous work dealing with linear configurations ${ }^{36}$ and confirmed later for nonlinear waveguides. $^{37}$ As shown in Figure $2 \mathrm{a}$, for $d=30 \mathrm{~nm}$, the fundamental optical modes propagating in the full structure are similar for TM and TE polarizations and resemble the guided mode profiles present without metal. To the contrary, for $d=$ $10 \mathrm{~nm}$, the TM mode clearly benefits from a strong localization at the chalcogenide/silica interface due to the plasmonic effect while the TE mode is nearly unaffected (Figure $2 \mathrm{~b}$ and section 4 of the Supporting Information). A 3-D distribution of the TM modes is depicted in Figures S1 and S2 of the Supporting Information. Simulations also show that a $10 \mathrm{~nm}$ buffer layer allows a decrease by a factor three of the propagation losses for the TM mode compared to a basic structure without silica buffer (see sections 3 and 4 of the Supporting Information). 

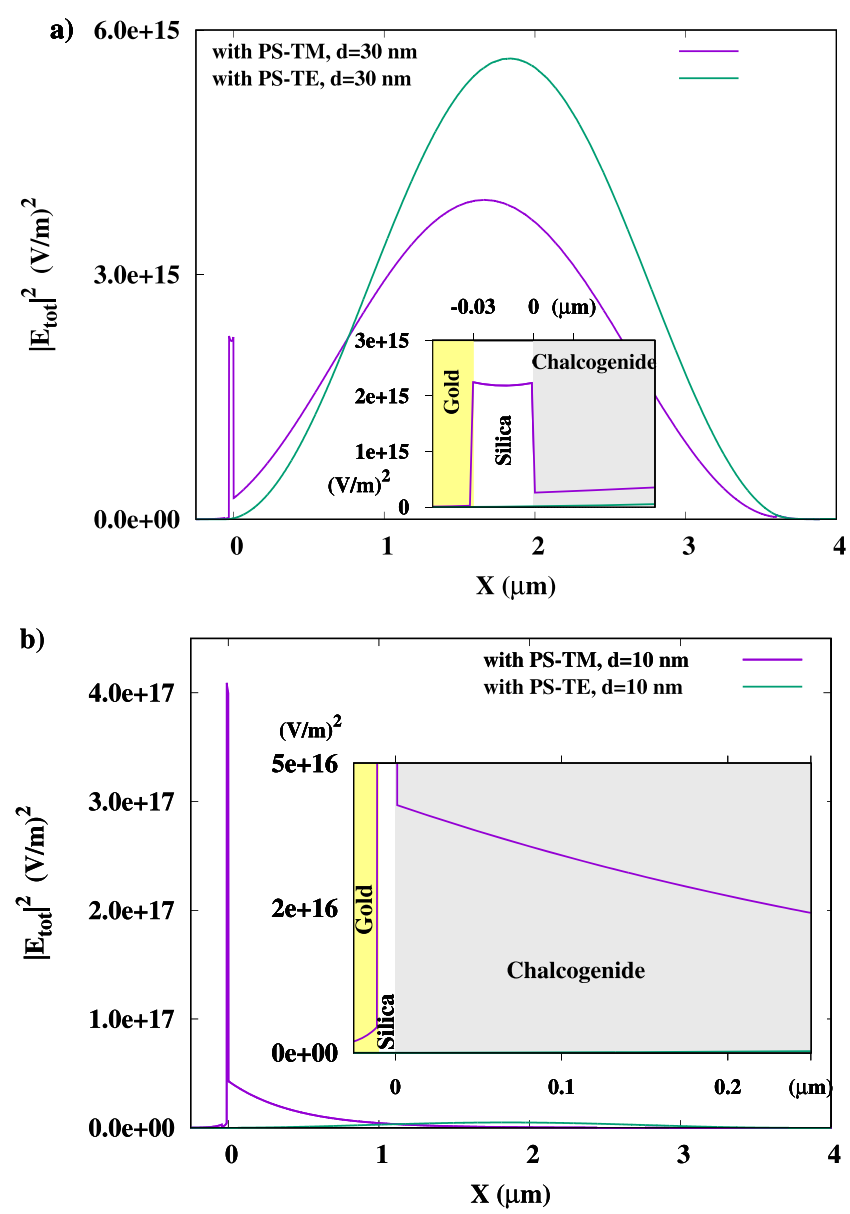

Figure 2. Calculated intensity profiles along the $X$-axis of the fundamental modes of same power $P_{\text {tot }}=513 \mathrm{~W}$ for TM (green curves) and TE (magenta curves) polarizations and zoom-in near the silica/gold interface (insets). $\mathrm{SiO}_{2}$ buffer thickness $d$ is equal to $30 \mathrm{~nm}$ (a) and $10 \mathrm{~nm}(\mathrm{~b})$.

The heterostructure fabrication was conducted in several steps. First, the $3.6 \mu \mathrm{m}$ thick $\mathrm{Ge}_{28.1} \mathrm{Sb}_{6.3} \mathrm{Se}_{65.6}$ chalcogenide layer is deposited by RF magnetron sputtering method on an oxidized silicon wafer. Then a $10 \mathrm{~nm}$ silica buffer layer, followed by a $32 \mathrm{~nm}$ gold layer, is formed on top to give the structure depicted in Figure $1 \mathrm{~b}$. While challenging, the deposition of the very thin $\mathrm{SiO}_{2}$ layer is operated by a sputtering deposition technique. A $32 \mathrm{~nm}$ gold nanolayer is chosen since it is thick enough to provide the plasmonic effect, while it can be deposited to form a uniform good quality layer (see section 1 of the Supporting Information for fabrication details). A cross section of the fabricated structure was analyzed by SEM (Figure 1c), confirming that the targeted and fabricated nanolayer thicknesses are consistent. As depicted in Figure 1d, the gold layer was then patterned to obtain either a rectangular or a staircase (Figure 1e) PS area. This arrangement provides the versatility to analyze several configurations by choosing the position of the launched beam along the $Y$-axis. Specifically, three different PS locations were considered as described in Figure $1 \mathrm{~d}$. The first one is a rectangular plasmonic pattern, $h=660 \mu \mathrm{m}$ long, located at the exit face of a $5.1 \mathrm{~mm}$ long sample (configuration I). The two other configurations correspond to a $5.5 \mathrm{~mm}$ long sample with a staircase PS with three different $h$ values equal to 100, 200, and $500 \mu \mathrm{m}$, present either $1 \mathrm{~mm}$ before the exit face or $1 \mathrm{~mm}$ after the entrance face to form configurations II and III, respectively. The input and output faces of the samples are formed by cleaving the samples.

\section{OPTICAL CHARACTERIZATIONS}

Characterizations are performed with an optical parametric oscillator (Chameleon OPO from Coherent) set to $1550 \mathrm{~nm}$ and emitting $200 \mathrm{fs}$ pulses at a repetition rate of $80 \mathrm{MHz}$ (Figure $3 \mathrm{a}$ and Supporting Information). The beam is elliptically shaped and focused with a cylindrical lens and a microscope objective to give a $4 \mu \mathrm{m}$ (fwhm) focal spot along the $X$-axis at the entrance of the waveguide, while a $31 \mu \mathrm{m}$ focal spot along the $Y$-axis is located $2 \mathrm{~mm}$ inside the sample in the linear regime (low intensity). Figure 3a shows the corresponding input beam profile at the entrance face of the waveguide. In the linear regime, the propagation beam is weakly diffracting along the $Y$-axis; as a consequence, the focal point position (along $Y$ ), relative to the PS location, has only a negligible influence in the studied behavior for the three configurations. Such a beam arrangement helps to prevent input facet damage in the high intensity regime. The beam profile at the output of the sample is imaged on a Vidicon camera with a $\times 10$ microscope objective. In the linear regime, the output beam reaches a fwhm of $41 \mu \mathrm{m}$ along the $Y$-axis (Figure 3a). Note that the imaging system was chosen to analyze the beam size variation along the $Y$-axis due to the selffocusing effect, but it was not adequate to resolve the much smaller beam $X$-dimension. Note also that a careful beam
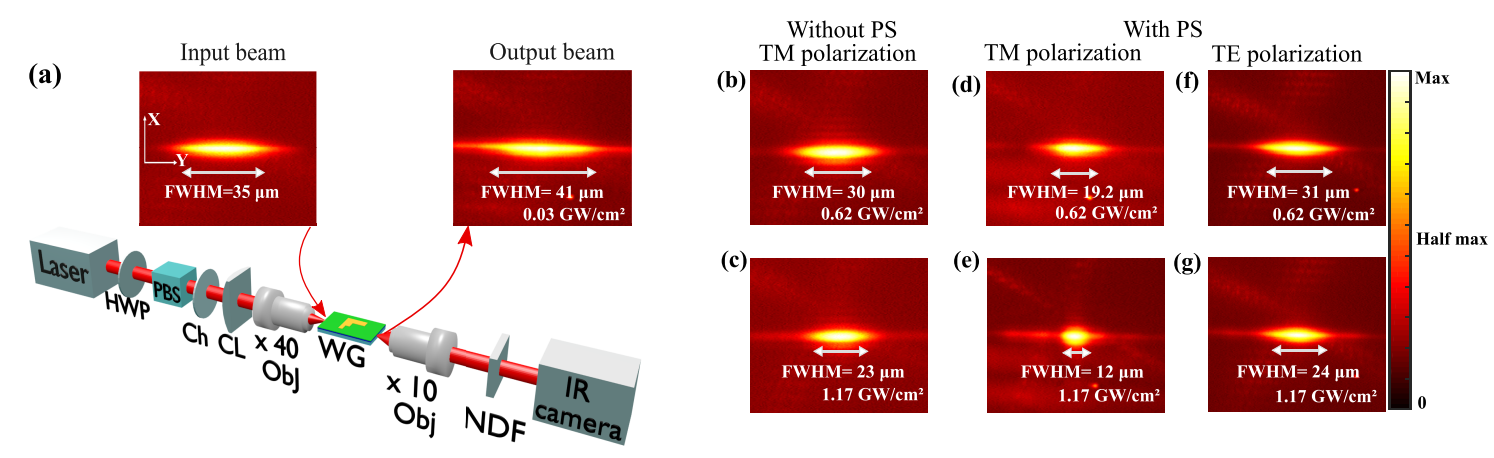

Figure 3. (a) Experimental setup for the observation of the self-confined nonlinear waves and intensity distribution of the input and output beams in the linear regime. HWP, half wave plate; PBS, polarizing beam splitter; Ch, chopper; CL, cylindrical lens; Obj, microscope objective; NDF, neutral density filters. $(b-g)$ Intensity distribution of self-focused output beams for two input intensities without PS (b, $c$ ), with PS corresponding to configuration I for TM polarization (d, e), and for TE polarization ( $f, g)$. 
(a)

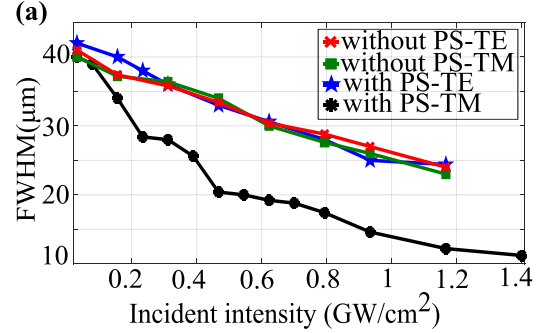

(b)

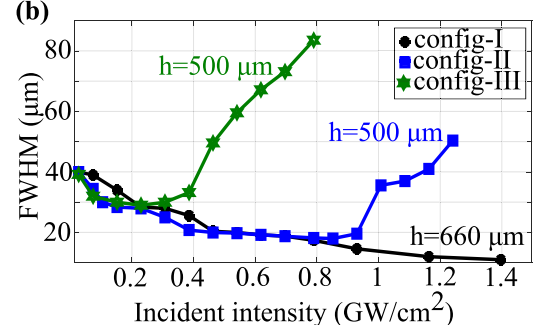

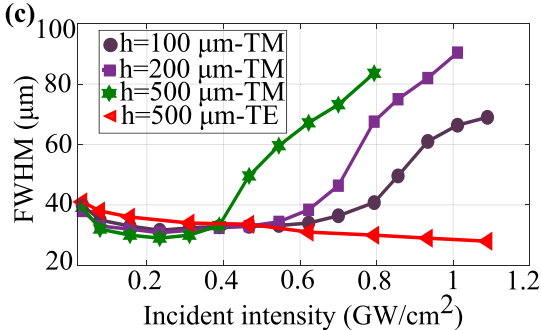

Figure 4. (a) Measured output beam fwhm as a function of input intensity for both TM and TE polarizations without PS and with a PS corresponding to configuration I. (b) Evolution of output beam fwhm as a function of input intensity for configurations I, II, and III for TM polarization. (c) Evolution of output beam fwhm for configuration III for PS lengths $h$ of 500, 200, and 100 $\mu \mathrm{m}$ for TM polarization and for $h$ of $500 \mu \mathrm{m}$ for TE polarization.

alignment is performed to maximize the beam overlap with the fundamental mode of the planar waveguide and thus avoid excitation of higher-order modes. Input and output powers are monitored by power meters to deduce the coupling efficiency, the peak power, and the waveguide transmission. In the linear regime a $21 \%$ waveguide transmission is measured for both $\mathrm{TM}$ and TE polarizations when light propagates away from the metallized area. This transmitted power is consistent with a coupling efficiency of $28 \%$ and propagation losses of about $0.19 \mathrm{~cm}^{-1}$.

To unambiguously reveal the influence of the plasmonic effect on the beam self-focusing, we first characterize the selftrapping behavior in the absence of metallic layer, referred as without PS, along the beam path. These preliminary experiments can thus be considered as a reference. As shown in refs 30 and 31, similar chalcogenide slab waveguides can support spatial solitons at near-infrared wavelengths. In the tested sample we observe that, for an input intensity of 0.62 $\mathrm{GW} / \mathrm{cm}^{2}$, a $30 \mu \mathrm{m}$ fwhm beam is obtained at the output face (Figure $3 \mathrm{~b}$ ). This size is close to the injected beam size of 31 $\mu \mathrm{m}$, which indicates that diffraction is compensated by the nonlinear Kerr self-focusing effect to form a spatial soliton. ${ }^{6}$ A stronger focusing effect occurs if the input intensity is further raised. For an intensity of $1.17 \mathrm{GW} / \mathrm{cm}^{2}$, a $23 \mu \mathrm{m}$ fwhm is obtained at the output (Figure $3 \mathrm{c}$ ). Note that the very same behavior is observed independently of the polarization for a configuration without PS (see Figure S9 in the Supporting Information).

We then shift laterally the sample so that a PS is present on the trajectory of the launched beam. Configuration I described in Figure $1 \mathrm{~d}$ is first considered with a PS length $h$ of $660 \mu \mathrm{m}$. In the linear regime, the presence of this metallic structure located at the end of the waveguide does not modify the observed output beam distribution. The linear transmission is however reduced due to additional propagation losses compared to the situation without the PS. We deduce that under the PS the attenuation is 0.57 and $28 \mathrm{~cm}^{-1}$ for TE and TM polarized light, respectively. The larger attenuation coefficient for the TM case is a first indication of the plasmonic effect. Subsequently, as the intensity is raised, a strong enhancement of the self-focusing behavior is observed at the output of the waveguide for TM waves. For instance, at an input intensity of $0.62 \mathrm{GW} / \mathrm{cm}^{2}$ (Figure $3 \mathrm{~d}$ ), the output beam is already much narrower (19 $\mu \mathrm{m}$ fwhm) than the injected one (31 $\mu \mathrm{m}$ fwhm), while a higher intensity of 1.17 $\mathrm{GW} / \mathrm{cm}^{2}$ leads to a very efficient trapping of the beam, as witnessed by an output beam of $12 \mu \mathrm{m}$ fwhm (Figure 3e). By comparing this result with the one obtained at the same intensity without PS (Figure 3c), we can deduce that the plasmonic enhanced nonlinear effect induced by the $660 \mu \mathrm{m}$ long PS focuses the beam from about 23 to $12 \mu \mathrm{m}$ fwhm. It is important to note that the exalted focusing is not observed for the TE polarization. Indeed, as shown in Figure $3 \mathrm{f}, \mathrm{g}$, the TE wave shows no improved self-focusing compared to the configuration without the PS (Figure $3 b, c)$. This polarization-dependent behavior is a characteristic feature of the plasmonic effect. To exclude the possible influence of thermally induced self-focusing in the nonlinear regime, experiments are repeated with a mechanical chopper positioned after the light source to diminish the average power to $40 \%$ while keeping the same peak power (see section 11 in the Supporting Information). No measurable change is observed compared to the behavior described in Figure 3, which excludes any significant role of a thermally induced nonlinearity.

The experimentally demonstrated striking self-focusing enhancement due to the plasmonic effect is also evident in Figure $4 \mathrm{a}$, where the measured output fwhm beam is plotted as a function of the input beam intensity for TE and TM polarizations with and without the PS. For the three arrangements for which the plasmonic effect is not present, that is, without or with PS for TE polarization and without PS for TM polarization, similar behaviors are observed. They are characterized by a gradual focusing with a nearly linear decrease of the beam width versus intensity. Remarkable features are observed for a TM polarized beam in the presence of a PS: (1) This plasmon-enhanced configuration always gives rise to an output fwhm beam smaller than for the other three configurations; (2) The focusing effect takes place at a faster pace for low intensity; (3) Three times less intensity is required compared to the cases without enhancement to obtain an output size of $31 \mu \mathrm{m}$; (4) The fwhm evolves with a nonlinear trend and tends to saturate to a strongly focused beam of $11 \mu \mathrm{m}$ fwhm at $1.4 \mathrm{GW} / \mathrm{cm}^{2}$.

To gain a better understanding of the disclosed self-trapped plasmonic wave and its strong focusing, additional configurations are tested. We first study the influence of the position of the PS. Figure $4 \mathrm{~b}$ compares the fwhm as a function of intensity for a $500 \mu \mathrm{m}$ long PS positioned either $1 \mathrm{~mm}$ before the exit face (Figure 1d, configuration II) or $1 \mathrm{~mm}$ after the input face (Figure 1d, configuration III) and the previously described case (configuration I) for a $660 \mu \mathrm{m}$ long PS. At very low intensity, the beam undergoes a similar free diffraction in the different samples, regardless of the PS location, precluding any linear beam distortion from the PS. As soon as the intensity is raised, the output beam focuses sharply in accordance with the Kerr lens effect present in the PS. 
However, when the intensity is increased above an intensity threshold a spreading of the beam is observed for PS located before the output face. The threshold is about 0.35 and 0.9 $\mathrm{GW} / \mathrm{cm}^{2}$ for PS positioned $1 \mathrm{~mm}$ after the entrance face (configuration III) or $1 \mathrm{~mm}$ before the exit face (configuration II), respectively. This behavior can once again be attributed to the strong localized self-focusing induced by the PS. Indeed, since the nonlinear focusing leads to an extremely narrow spot, the beam diffraction cannot be compensated anymore by the weaker nonlinearity present after the PS and beam diffraction dominates. The diffracted beam is narrower in configuration II since the PS is only $1 \mathrm{~mm}$ from the output face and diffraction then occurs on a shorter distance compared to configuration III. The numerical model presented later in the manuscript confirms this assumption and gives a clearer picture of the phenomena. The observed higher input intensity threshold when the PS is further to the input face (Figure $4 b$, configuration II) is also expected. Indeed, diffraction overtakes self-focusing when the beam reaching the PS exceeds a threshold intensity. To attain this threshold, a higher input intensity is necessary when the PS is further to the input face to compensate for propagation losses occurring along the input-PS distance.

To analyze the impact of the length $h$ of the PS, experiments are also conducted for PS lengths of 100 and $200 \mu \mathrm{m}$ for configuration III (Figure 1d,e). The results are presented in Figure 4c, along with measurements made for $h=500 \mu \mathrm{m}$ and for the enhancement-free TE polarization. As the intensity increases, the PS self-focusing enhancement gives a minimum fwhm value before the spatial spreading occurs. The shorter the PS, the weaker the nonlinear focusing effect. The minimum beam size is reached at an intensity of about $0.24 \mathrm{GW} / \mathrm{cm}^{2}$, giving an output fwhm of 31.6, 30.7, and $29 \mu \mathrm{m}$ for 100, 200, and $500 \mu \mathrm{m}$ PS length, respectively. As the intensity is increased further, beam spreading becomes dominant. This assumption of a beam widening dictated by diffraction authorizes an indirect evaluation of the spot size reached at the exit of the PS. Indeed, from the Gaussian beam theory, a 13 $\mu \mathrm{m}$ fwhm spot is expected to give a $70 \mu \mathrm{m}$ fwhm over $4 \mathrm{~mm}$ distance, as observed in Figure 4c for TM-polarized beams for configuration III for an input intensity of $1.1 \mathrm{GW} / \mathrm{cm}^{2}$. This predicted spot size is consistent with the $12 \mu \mathrm{m}$ fwhm direct measurement shown in Figure $3 \mathrm{e}$ for configuration $\mathrm{I}$ for an input intensity of $1.17 \mathrm{GW} / \mathrm{cm}^{2}$. From the beam widening obtained in the high intensity regime in Figure 4c, we can also conclude that self-focusing is more efficient in long PS structures. For instance, a $70 \mu \mathrm{m}$ output fwhm resulting from an assumed $13 \mu \mathrm{m}$ fwhm self-focused spot is induced by an input intensity of $0.7 \mathrm{GW} / \mathrm{cm}^{2}$ for a $500 \mu \mathrm{m}$ long PS, while a $1.1 \mathrm{GW} / \mathrm{cm}^{2}$ is requested for a $100 \mu \mathrm{m}$ long PS (Figure $4 \mathrm{c}$ ). It is remarkable that even a PS as short as $100 \mu \mathrm{m}$ can induce a strong focusing.

The analysis of the output beam profile provides crucial additional information that was not revealed by the fwhm measurements. In Figure 5, the output beam profile is plotted for six intensity values for a $500 \mu \mathrm{m}$ long PS corresponding to configuration II. The efficient focusing effect previously described forms at first a smooth profile beam with a weak pedestal (Figure 5b). This beam-cleaning feature is a characteristic of a spatial soliton regime. It thus may indicate that an intensity of $0.5 \mathrm{GW} / \mathrm{cm}^{2}$ triggers this regime for configuration II. As the intensity is further raised, the beam then enlarges and develops symmetric side lobes on both sides
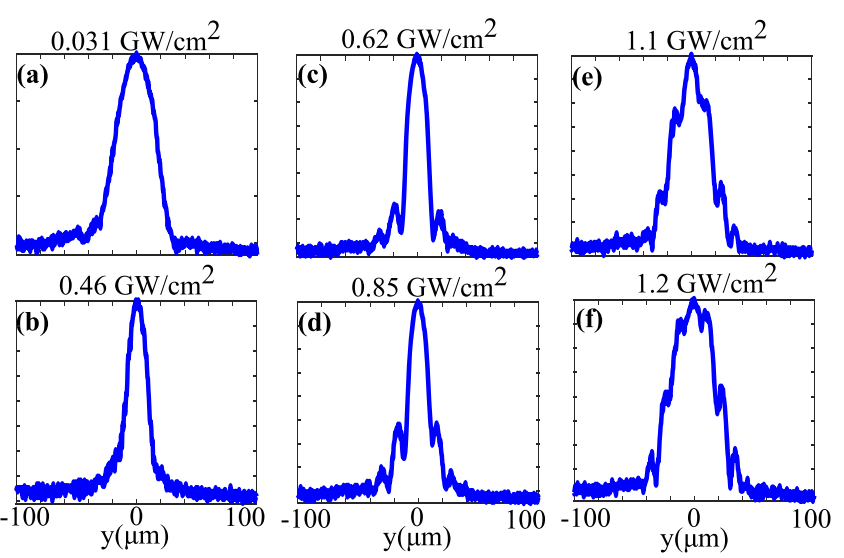

Figure 5. Measured output beam profile evolution as a function of incident intensity for configuration II, TM polarization, $h=500 \mu \mathrm{m}$.

of the main peak (Figure $5 \mathrm{c}-\mathrm{f}$ ). To gain more insight into the observed enhanced self-focusing phenomena, a numerical model presented in the following section has been developed.

\section{SIMULATIONS}

The numerical scalar model is based on a modified spatial nonlinear Schrödinger equation (SNLSE). This scalar model describes the transverse field profile along the $Y$-axis and its evolution versus propagation along the $Z$-axis. ${ }^{6,38}$ It is adapted to take into account the mode field profile along the $X$-axis that is dependent on polarization, power or location along propagation in the full structure. An example of this $x$ dependence is illustrated in Figure 2 for TE and TM modes. The field $x$-dependency does not explicitly appear in the SNLSE but is considered indirectly through the $z$-dependent modal properties (effective nonlinearity and propagation constants) that appear in the SNLSE. These modal parameters are obtained from the FEM-based simulations of the fundamental TE and TM modes associated with the different sections of the full structure ${ }^{34,35}$ (see sections $3-5$ of the Supporting Information).

We consider that the structure is stepwise constant along the $Z$-axis. In each discretized segment of the propagation, the fundamental mode is first determined using our FEM mode solver mentioned above. The mode $X Y$-distribution is thus determined together with its propagation constant in each segment.

It is worth to remind that only the transverse dimension along $y$ is usually considered for Kerr spatial solitons in slab waveguides studies. ${ }^{38,39}$ To also take into account the influence of the spatial field $x$-profile, we start from the nonlinear evolution equation where both $x$ and $y$ variables are considered. To obtain the final SNLSE involving only the transverse coordinate $y$ and the propagation direction coordinate $z$, we proceed by analogy with ref 40 , where the NLSE is derived in the temporal domain using a spatial integration in the cross section of the waveguide. In our study we limit the spatial integration on the nonlinear region of the $X$-axis only (denoted NL layer below) and we keep the dependency along the $Y$-axis. $\mathbf{E}(\mathbf{r}, t)$ being the electric field of the propagating wave, we also renormalize the investigated amplitude denoted by $\tilde{A}(y, z)$ in order to get directly the optical intensity $I$ when one takes its modulus squared: 


$$
\begin{aligned}
& \mathbf{E}(\mathbf{r}, t)=\frac{1}{2} \hat{\mathbf{u}}\left(F(x) A(y, z) \exp \left(-\mathrm{i}\left(\omega_{0} t-\beta_{0} z\right)\right)+\mathrm{cc}\right) \\
& |\tilde{A}(y, z)|^{2}=|A(y, z)|^{2} \frac{2}{\varepsilon_{0} n c}
\end{aligned}
$$

where cc means the complex conjugate and $\hat{\mathbf{u}}$ is a unitary vector in the waveguide cross section giving the studied polarization. $\tilde{A}(y, z)$ obeys the following SNLSE:

$$
\begin{aligned}
\frac{\partial \tilde{A}(y, z)}{\partial z}= & \frac{i}{2 \beta} \frac{\partial^{2} \tilde{A}(y, z)}{\partial y^{2}}-\frac{\alpha}{2} \tilde{A}(y, z)-\frac{\alpha_{2}}{2}|\tilde{A}(y, z)|^{2} \tilde{A}(y, z) \\
& +\frac{i \omega_{0} n_{2}}{c} \eta_{x}^{1 D}|\tilde{A}(y, z)|^{2} \tilde{A}(y, z)
\end{aligned}
$$

with

$$
\eta_{x}^{1 D} \equiv \int_{\text {NL_layer }} F(x)^{4} \mathrm{~d} x / \int_{\text {NL_layer }} F(x)^{2} \mathrm{~d} x
$$

The 1D normalized profile $F(x)$ is obtained from the 2D nonlinear profiles of the electromagnetic field of the fundamental mode in the considered segment computed with the FEM modal method. $F(x)$ is the $x$ profile obtained at $y=0$ and normalized according to the formula $\int_{-\infty}^{+\infty} F(x)^{2} \mathrm{~d} x=$ 1. The one-dimensional integrals defining $\eta_{x}^{1 D}$ are computed only over the nonlinear region. These integrals along with the propagation constant $\beta$ and the full field profiles are computed using our FEM mode solver. $\eta_{x}^{1 D}$ is called the opto-geometrical nonlinear factor. Before pursuing the description of the $\eta_{x}^{1 D}$ quantity, it is needed to explain how the experimental value of the material nonlinear coefficient $n_{2}$ has been obtained. In several cases, its value is derived from nonlinear propagation simulations using the 2D SNLSE where the transverse fwhm is computed and then compared to the measured ones as we have done in ref 31 . However, the SNLSE in the latter reference did not take into account the field $x$-profile and consequently the factor $\eta_{x}^{1 D}$ was missing. This factor $\eta_{x}^{1 D}$ that only appears in the last term of (3) is similar to the $\eta$ parameter described in the derivation of the nonlinear Schrödinger equation in the temporal domain for optical fibers by formula (5.26) in Okamoto's book. ${ }^{41}$ The main difference is that we integrate only along the $\mathrm{x}$-dimension corresponding to the thickness of the nonlinear layer while the spatial integration is realized on the full $2 \mathrm{D}$ cross section of the $\mathrm{HE}_{11}$ mode of a circular step-index fiber in. ${ }^{41}$ The values of $\eta_{x}^{1 D}$ are computed using the nonlinear fixed power algorithm described in ref 35 for the studied structures. They are given in Table 1 in the Supporting Information, for the fundamental TE and TM modes with and without the PS. The TE mode without PS and TM mode without PS correspond to the cases used to evaluate the nonlinear coefficient $n_{2}$ in ref 31 . As a result, the previously provided $n_{2}$ takes into account the mode related quantity $\eta_{x}^{1 D}$ for the TE and TM cases without PS, its value is numerically evaluated to approximately 0.4 . For the TM mode with PS, $\eta_{x}^{1 D}$ is between 3.15 and 3.51 times higher than for the other cases. This ratio $\eta_{x}^{1 D}$ (TM with PS) $/ \eta_{x}^{1 D}$ (TM without PS) is the key parameter of the plasmonic nonlinear reinforcement and will be called the effective nonlinearity enhancement factor. It slightly increases with the mode power, as shown in Table 1 in the Supporting Information. Consequently, the model gives an effective nonlinearity enhancement for the TM mode with PS at least 3 -fold the ones without PS. We, however, found that an effective nonlinearity improvement of 8 has to be set in order to quantitatively predict the above-described experimental results. We mainly attribute this discrepancy to our modeling approach and also to the potential nonlinear contribution of gold, as discussed later. Note also that this enhancement factor is low compared to plasmonic studies involving standing waves or localized resonances based on metallic nanostructures coupled to an electromagnetic field that can provide an enhancement factor above $100 .^{2,5}$ It is important to point out that our experiment instead involves propagating plasmonic waves. In ref 42 , dealing with a quite similar configuration, but for a structure based on $\mathrm{CS}_{2}$ and silver nanoparticles, the authors computed an enhancement factor of 2 , which is in the same range as ours. We would like to emphasize that, apart from this enhancement factor, other simulation parameters, notably linear and nonlinear absorption coefficients, are the ones determined experimentally in the previous sections. It is worth pointing out that the developed model also gives results in accordance with our experimental results for Gaussian beams propagating both in linear and nonlinear regimes in the slab nonlinear waveguide. The observed spatial soliton propagation is notably predicted. The numerical implementation of the resolution of the nonlinear propagation equation is detailed in section 9 of the Supporting Information. Quantitative comparisons between the numerical predictions and the experimental observations for the plasmon-enhanced self-trapping are presented in the following section.

\section{DISCUSSION}

Figure 6a presents the calculated profile along the $Y$-axis of the beam intensity versus propagation inside the full structure for the TM polarization for configuration II with $h=500 \mu \mathrm{m}$ at $1.25 \mathrm{GW} / \mathrm{cm}^{2}$ input intensity. This emblematic case shown in Figure 6 a reveals several remarkable characteristics that give more insight into the behavior unveiled experimentally. First, we see that the beam self-focuses along the first $2 \mathrm{~mm}$ propagation and then becomes nearly invariant before converging dramatically to a highly focused central peak as it travels in the $500 \mu \mathrm{m}$ long PS region localized between the dashed lines. Finally, as the beam leaves this highly nonlinear PS region, diffraction overtakes the self-focusing effect (see Figure S6 in the Supporting Information for the results for the same configuration at $15 \mathrm{MW} / \mathrm{cm}^{2}$ input intensity). It is important to note that the numerical model not only predicts the spreading once the beam exits the PS, but it also depicts the symmetric lateral peaks observed experimentally (Figure $5)$. These peaks are generated in the last part of the PS for intensities above $0.6 \mathrm{GW} / \mathrm{cm}^{2}$, a value in fair agreement with the experiments. These specific profiles also play a role in the beam spreading observed at high intensity for configurations II and III (see sections 6 and 7 of the Supporting Information for details). Although spatial modulation instability is known to induce beam breakup of an initial noisy broad beam propagating in Kerr nonlinear media, it does not fit to the present phenomenom. Indeed, the measured intensity dependence of the generated spatial frequency does not follow the scaling law of modulation instability ${ }^{43,44}$ (see section 13 in the Supporting Information). Moreover, large amplitude spatial noise added in the present simulations does not give rise to the observed multipeaks unless the plasmon-enhanced nonlinearity is present. We think the phenomenon revealed here is a combination of the beam breakup described in refs 45-47, induced at the interface between two dielectric regions of high 

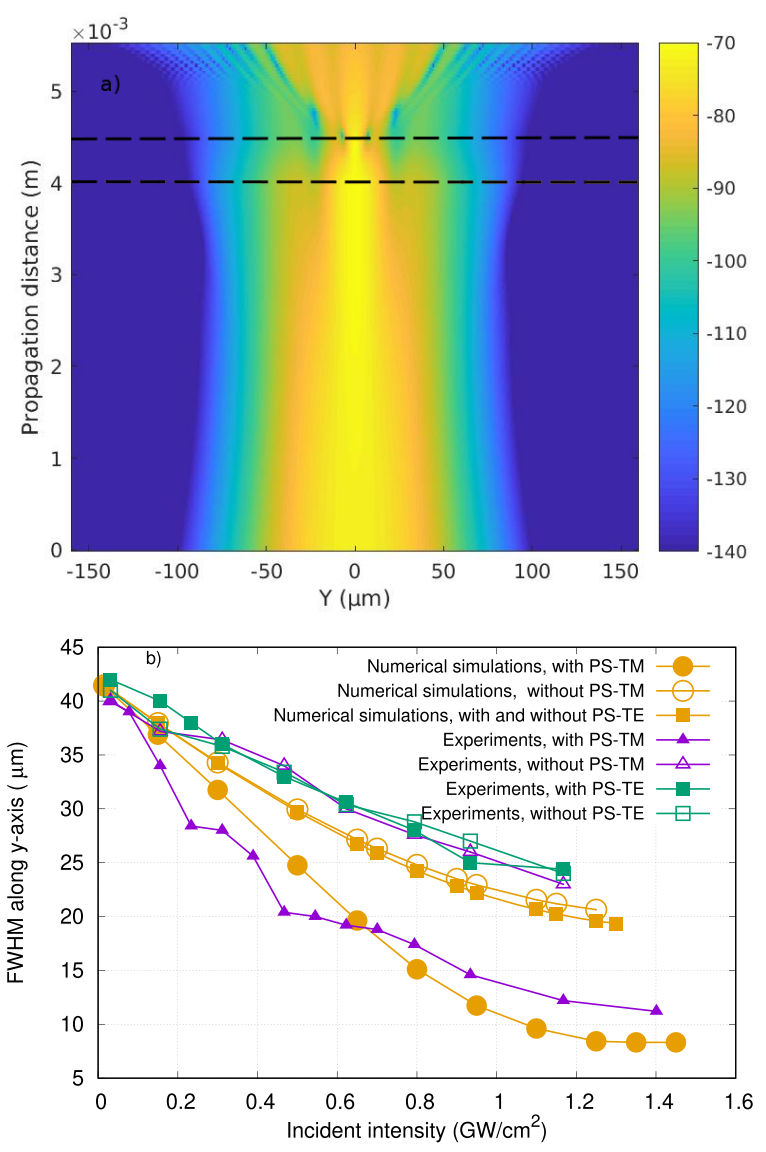

Figure 6. Numerical results. (a) Color map in log scale of the beam intensity evolution along the $Y$-axis vs propagation inside the full structure for TM polarization for configuration II, with $h=500 \mu \mathrm{m}$ in the nonlinear regime (input intensity of $1.25 \mathrm{GW} / \mathrm{cm}^{2}$ ). The dashed lines represent the limits of the PS. (b) Comparison of the computed and measured fwhm for configuration $\mathrm{I}$ as a function of the input beam intensity. Note that, at this scale, there is no visible difference between the simulation results for TE polarization with or without PS (filled orange squares).

and low Kerr-type nonlinearities and of the symmetric lateral peaks generated for intensities above the soliton regime.

The comparison between the measured and the computed fwhm as a function of the incident intensity, obtained for configuration I for TE and TM polarizations, is provided in Figure $6 \mathrm{~b}$. The nonlinear behavior is in agreement with the observations. Numerical results obtained for TE polarization with and without PS are represented by the same calculated points (filled orange squares) since effective nonlinearity enhancement and losses are fully similar for both cases. The model applied to a TM wave in the absence of PS (open orange circles) predicts an equivalent behavior. These three cases mimics fairly well the corresponding experimental data. More importantly, the simulations for TM polarization with PS (filled orange circles) nicely follow the experimental measurements. Note that the saturation of the self-focusing effect appearing experimentally at high power is also reproduced by the simulations. This proves that the plasmonic effect due to the PS is able to quantitatively explain the observed large focusing enhancement. The validity of our numerical model is further confirmed by comparison with experimental results from configurations II and III. The influence of the PS length and the appearance of beam widening at high power is notably clearly depicted (see section 6 in the Supporting Information).

Quantitative discrepancies between theory and experiments, however, remain. The main one is the effective nonlinearity enhancement factor computed from FEM that is 2.5 times weaker than the one used in the SNLSE simulations to obtain the best fit with experimental results. First, some limitations of the model can be invoked: it is based on a scalar SNLSE, while the true problem is a vector one; paraxial approximations and the calculation of the effective nonlinearity are not fully valid when the beam profile rapidly transforms in the PS. Second, the considered instantaneous response of the Kerr effect could be questioned in the femtosecond regime. ${ }^{48}$ Third, there are some uncertainties on the values of the nonlinear coefficients $n_{2}$ and $\alpha_{2}$ of this uncommon chalcogenide composition. ${ }^{49}$ Finally, the gold nanolayer permittivity, thickness uniformity, and its potential nonlinearity ${ }^{50-52}$ can also be a reason for discrepancies between simulation results and measurements.

\section{CONCLUSION}

In summary, a compelling spatial confinement of a guided beam observed only for TM polarization, in a Kerr plasmonic structure, has been demonstrated experimentally. The designed structure consists of a slab waveguide made of a highly nonlinear chalcogenide layer covered with nanolayers of silica and gold. The thickness of the silica layer has been tailored using FEM modal simulations to benefit from an enhanced self-focusing due to the plasmonic part of the wave while limiting the detrimental effect of propagation losses induced by the metal. Experimental observations are confirmed by numerical predictions obtained using a tailored model of nonlinear propagation combining FEM modal results and SNLSE. This experimental proof validates the concept of nonlinear spatial optical self-trapping in guided optics enhanced by a plasmonic effect and paves the way to the formation of shape preserving plasmon-soliton waves. The dramatic nonlinear beam reshaping induced over a distance of a few hundred microns at moderate light power opens up new perspectives for nonlinear integrated plasmonic devices. Further investigations such as pump-probe experiments are foreseen to complete the quantitative description of the observed phenomena and to assess the potential for all-optical signal processing of these nonlinear structures.

\section{ASSOCIATED CONTENT}

\section{Supporting Information}

The Supporting Information is available free of charge at https://pubs.acs.org/doi/10.1021/acsphotonics.0c00906.

There are 13 sections: Structure fabrication; Experimental characterizations; Simulations: modal analysis with the finite element method; Details of the nonlinear mode profiles; Impact of parameter variability for the nonlinear mode simulations of configuration I; Complementary simulation results for configuration II; Complementary results for configurations I, II, and III; Discussion on the limitations of the SNLSE; Numerical implementation of the nonlinear propagation equation; Experimental results: TE polarization vs TM polarization without PS; Test of thermal effects; Complementary discussion on the solitonic nature of the selftrapped waves; Test of the spatial modulation (PDF) 


\section{AUTHOR INFORMATION}

\section{Corresponding Authors}

Gilles Renversez - Aix-Marseille Univ, CNRS, Centrale Marseille, Institut Fresnel, 13013 Marseille, France; ๑ orcid.org/0000-0002-7184-2665; Email: gilles.renversez@ univ-amu.fr

Mathieu Chauvet - FEMTO-ST Institute, CNRS, University of Bourgogne Franche-Comte, 25030 Besançon, France; Email: mathieu.chauvet@univ-fcomte.fr

\section{Authors}

Tintu Kuriakose - FEMTO-ST Institute, CNRS, University of Bourgogne Franche-Comté, 25030 Besançon, France

Virginie Nazabal - Univ Rennes, CNRS, ISCR (Institut des Sciences Chimiques de Rennes) - UMR 6226, 35000 Rennes, France; (1) orcid.org/0000-0002-0113-3935

Mahmoud M. R. Elsawy - Aix-Marseille Univ, CNRS, Centrale Marseille, Institut Fresnel, 13013 Marseille, France

Nathalie Coulon - Univ Rennes, CNRS, IETR (Institut d'Electronique et de Technologies du numérique) - UMR 6164, 35000 Rennes, France

Petr Němec - Department of Graphic Arts and Photophysics, Faculty of Chemical Technology, University of Pardubice, 53210 Pardubice, Czech Republic

Complete contact information is available at: https://pubs.acs.org/10.1021/acsphotonics.0c00906

\section{Notes}

The authors declare no competing financial interest.

\section{ACKNOWLEDGMENTS}

The authors acknowledge the financial support of the Région Franche-Comte, of Aix-Marseille University, and of the Czech Science Foundation (GACR Project 19-24516S). This work was also partially supported by the FEMTO-ST MIMENTO technological facilities.

\section{REFERENCES}

(1) Cunningham, S. L.; Maradudin, A. A.; Wallis, R. F. Effect of a charge layer on the surface-plasmon-polariton dispersion curve. Phys. Rev. B 1974, 10, 3342-3355.

(2) Maier, S. A. Plasmonics: Fundamentals and Applications; Springer, 2007.

(3) Cui, T. J.; Liu, R.; Smith, D. R. In Metamaterials: Theory, Design, and Applications, 1st ed.; Cui, T. J., Smith, D., Liu, R., Eds.; Springer: U.S., 2010.

(4) Liberal, I.; Engheta, N. Near-zero refractive index photonics. Nat. Photonics 2017, 11, 149-158.

(5) Kauranen, M.; Zayats, A. V. Nonlinear plasmonics. Nat. Photonics 2012, 6, 737-748.

(6) Trillo, S., Torruellas, W., Eds. In Spatial Solitons; Optical Sciences; Springer, 2001.

(7) Segev, M.; Valley, G. C.; Crosignani, B.; DiPorto, P.; Yariv, A. Steady-State Spatial Screening Solitons in Photorefractive Materials with External Applied Field. Phys. Rev. Lett. 1994, 73, 3211-3214.

(8) Fazio, E.; Renzi, F.; Rinaldi, R.; Bertolotti, M.; Chauvet, M.; Ramadan, W.; Petris, A.; Vlad, V. I. Screening-photovoltaic bright solitons in lithium niobate and associated single-mode waveguides. Appl. Phys. Lett. 2004, 85, 2193-2195.

(9) Peccianti, M.; Conti, C.; Assanto, G.; De Luca, A.; Umeton, C. Routing of anisotropic spatial solitons and modulational instability in liquid crystals. Nature 2004, 432, 733-737.

(10) Aitchison, J. S.; Weiner, A. M.; Silberberg, Y.; Oliver, M. K.; Jackel, J. L.; Leaird, D. E.; Vogel, E. M.; Smith, P. W. E. Observation of spatial optical solitons in a nonlinear glass waveguide. Opt. Lett. 1990, 15, 471.

(11) Suntsov, S.; Makris, K. G.; Christodoulides, D. N.; Stegeman, G. I.; Haché, A.; Morandotti, R.; Yang, H.; Salamo, G.; Sorel, M. Observation of Discrete Surface Solitons. Phys. Rev. Lett. 2006, 96, 063901.

(12) Stegeman, G. I.; Wright, E. M.; Finlayson, N.; Zanoni, R.; Seaton, C. T. Third order nonlinear integrated optics. J. Lightwave Technol. 1988, 6, 953-970.

(13) Martellucci, S.; Chester, A. N.; Bertolotti, M. Advances in Integrated Optics, reprint of the original 1st ed., 1994 ed.; Springer, 2012.

(14) Agranovich, V. M.; Babichenko, V. S.; Chernyak, V. Y. Nonlinear surface polaritons. JETP Lett. 1980, 32, 512-515.

(15) Tomlinson, W. J. Surface wave at a nonlinear interface. Opt Lett. 1980, 5, 323-325.

(16) Ariyasu, J.; Seaton, C. T.; Stegeman, G. I.; Maradudin, A. A.; Wallis, R. F. Nonlinear surface polaritons guided by metal films. J. Appl. Phys. 1985, 58, 2460-2466.

(17) Akhmediev, N. Novel class of nonlinear surface waves: asymmetric modes in a symmetric layered structure. Sov. Phys. JETP 1982, 56, 299-303.

(18) Feigenbaum, E.; Orenstein, M. Plasmon-soliton. Opt. Lett. 2007, 32, 674.

(19) Davoyan, A. R.; Shadrivov, I. V.; Kivshar, Y. S. Self-focusing and spatial plasmon-polariton solitons. Opt. Express 2009, 17, 2173221737.

(20) Bliokh, K. Y.; Bliokh, Y. P.; Ferrando, A. Resonant plasmonsoliton interaction. Phys. Rev. A: At., Mol., Opt. Phys. 2009, 79, 41803.

(21) Walasik, W.; Nazabal, V.; Chauvet, M.; Kartashov, Y.; Renversez, G. Low-power plasmon-soliton in realistic nonlinear planar structures. Opt. Lett. 2012, 37, 4579-4581.

(22) Walasik, W.; Renversez, G.; Kartashov, Y. V. Stationary plasmon-soliton waves in metal-dielectric nonlinear planar structures: Modeling and properties. Phys. Rev. A: At., Mol., Opt. Phys. 2014, 89, 023816 .

(23) Walasik, W.; Renversez, G. Plasmon-soliton waves in planar slot waveguides. I. Modeling. Phys. Rev. A: At., Mol., Opt. Phys. 2016, 93, 013825

(24) Walasik, W.; Renversez, G.; Ye, F. Plasmon-soliton waves in planar slot waveguides. II. Results for stationary waves and stability analysis. Phys. Rev. A: At., Mol., Opt. Phys. 2016, 93, 013826.

(25) Marini, A.; Biancalana, F. Ultrashort Self-Induced Transparency Plasmon Solitons. Phys. Rev. Lett. 2013, 110, 243901.

(26) Smirnova, D. A.; Shadrivov, I. V.; Smirnov, A. I.; Kivshar, Y. S. Dissipative plasmon-solitons in multilayer graphene. Laser \& Photonics Reviews 2014, 8, 291-296.

(27) Nesterov, M. L.; Bravo-Abad, J.; Nikitin, A. Y.; García-Vidal, F. J.; Martin-Moreno, L. Graphene supports the propagation of subwavelength optical solitons. Laser \& Photonics Reviews 2013, 7 , L7-L11.

(28) Pusch, A.; Shadrivov, I. V.; Hess, O.; Kivshar, Y. S. Self-focusing of femtosecond surface plasmon polaritons. Opt. Express 2013, 21, $1121-1127$

(29) Zakery, A.; Elliott, S. R. Optical Nonlinearities in Chalcogenide Glasses and their Applications; Springer, 2007.

(30) Chauvet, M.; Fanjoux, G.; Huy, K. P.; Nazabal, V.; Charpentier, F.; Billeton, T.; Boudebs, G.; Cathelinaud, M.; Gorza, S.-P. Kerr spatial solitons in chalcogenide waveguides. Opt. Lett. 2009, 34, 1804-1806.

(31) Kuriakose, T.; Baudet, E.; Halenkovič, T.; Elsawy, M. M.; Němec, P.; Nazabal, V.; Renversez, G.; Chauvet, M. Measurement of ultrafast optical Kerr effect of $\mathrm{Ge}-\mathrm{Sb}$-Se chalcogenide slab waveguides by the beam self-trapping technique. Opt. Commun. 2017, 403, 352357.

(32) Nazabal, V.; Charpentier, F.; Adam, J.-L.; Nemec, P.; Lhermite, H.; Brandily-Anne, M.-L.; Charrier, J.; Guin, J.-P.; Moreac, A. Sputtering and Pulsed Laser Deposition for Near- and Mid-Infrared 
Applications: A Comparative Study of $\mathrm{Ge}_{25} \mathrm{Sb}_{10} \mathrm{~S}_{65}$ and $\mathrm{Ge}_{25} \mathrm{Sb}_{10} \mathrm{Se}_{65}$. Int. J. Appl. Ceram. Technol. 2011, 8, 990.

(33) Olivier, M.; Tchahame, J.; Němec, P.; Chauvet, M.; Besse, V.; Cassagne, C.; Boudebs, G.; Renversez, G.; Boidin, R.; Baudet, E.; Nazabal, V. Structure, nonlinear properties, and photosensitivity of $(\mathrm{GeSe} 2) 100-\mathrm{x}(\mathrm{Sb} 2 \mathrm{Se} 3) \mathrm{x}$ glasses. Opt. Mater. Express 2014, 4, 525540.

(34) Elsawy, M. M. R.; Renversez, G. Study of plasmonic slot waveguides with a nonlinear metamaterial core: semi-analytical and numerical methods. J. Opt. 2017, 19, 075001.

(35) Elsawy, M. M. R.; Renversez, G. Exact calculation of the nonlinear characteristics of $2 \mathrm{D}$ isotropic and anisotropic waveguides. Opt. Lett. 2018, 43, 2446-2449.

(36) Oulton, R. F.; Sorger, V. J.; Genov, D. A.; Pile, D. F. P.; Zhang, $\mathrm{X}$. A hybrid plasmonic waveguide for subwavelength confinement and long-range propagation. Nat. Photonics 2008, 2, 496-500.

(37) Elsawy, M. M. R.; Renversez, G. Improved nonlinear slot waveguides using dielectric buffer layers: properties of TM waves. Opt. Lett. 2016, 41, 1542-1545.

(38) Powers, P. E.; Haus, J. W. Fundamentals of Nonlinear Optics, 2nd ed.; CRC Press, 2017.

(39) Kivshar, Y. S.; Agrawal, G. P. Optical Solitons, From Fibers to Photonic Crystals; Academic Press, 2003.

(40) Agrawal, G. P. Nonlinear Fiber Optics, 5th ed.; Academic Press, 2013.

(41) Okamoto, K. In Fundamentals of Optical Waveguides, 2nd ed.; Okamoto, K., Ed.; Academic Press, 2006; pp 209-259.

(42) Kohlgraf-Owens, D. C.; Kik, P. G. Numerical study of surface plasmon enhanced nonlinear absorption and refraction. Opt. Express 2008, 16, 10823-10834.

(43) Malendevich, R.; Jankovic, L.; Stegeman, G.; Aitchison, J. S. Spatial modulation instability in a Kerr slab waveguide. Opt. Lett. 2001, 26, 1879-1881.

(44) Lin, Y.-Y.; Lee, R.-K.; Kivshar, Y. S. Transverse instability of transverse-magnetic solitons and nonlinear surface plasmons. Opt. Lett. 2009, 34, 2982-2984.

(45) Aceves, A. B.; Moloney, J. V.; Newell, A. C. Theory of lightbeam propagation at nonlinear interfaces. II. Multiple-particle and multiple-interface extensions. Phys. Rev. A: At., Mol., Opt. Phys. 1989, 39, $1828-1840$.

(46) Sánchez-Curto, J.; Chamorro-Posada, P.; McDonald, G. S. Helmholtz bright and black soliton splitting at nonlinear interfaces. Phys. Rev. A: At., Mol., Opt. Phys. 2012, 85, 013836.

(47) Aleshkevich, V. A.; Kartashov, Y. V.; Zelenina, A. S.; Vysloukh, V. A.; Torres, J. P.; Torner, L. Eigenvalue control and switching by fission of multisoliton bound states in planar waveguides. Opt. Lett. 2004, 29, 483-485.

(48) Cambournac, C.; Maillotte, H.; Lantz, E.; Dudley, J. M.; Chauvet, M. Spatiotemporal behavior of periodic arrays of spatial solitons in a planar waveguide with relaxing Kerr nonlinearity. J. Opt. Soc. Am. B 2002, 19, 574-585.

(49) Boudebs, G.; Cherukulappurath, S.; Leblond, H.; Troles, J.; Smektala, F.; Sanchez, F. Experimental and theoretical study of higher-order nonlinearities in chalcogenide glasses. Opt. Commun. 2003, 219, 427-433.

(50) Boyd, R. W.; Shi, Z.; De Leon, I. The third-order nonlinear optical susceptibility of gold. Opt. Commun. 2014, 326, 74-79.

(51) Qian, H.; Xiao, Y.; Liu, Z. Giant Kerr response of ultrathin gold films from quantum size effect. Nat. Commun. 2016, 7, 1-6.

(52) Tuniz, A.; Weidlich, S.; Schmidt, M. A. Effectively Single-Mode Self-Recovering Ultrafast Nonlinear Nanowire Surface Plasmons. Phys. Rev. Appl. 2018, 9, 044012. 